\section{EFFECT ON THE EARLY EMBRYO OF AGENTS ADMINISTERED TO THE MOTHER}

BY

\section{Cecilia LUTWAK-ManN, M.D., Ph.D.}

AND

\author{
MARY F. HAY, Ph.D., B.Sc.
}

Agricultural Research Council Unit of Reproductive Physiology and Biochemistry, University of Cambridge

\section{[With Special Plate]}

In some recent investigations into the physiology and biochemistry of the pre-implantation rabbit embryo (blastocyst), we have carried out numerous experiments on the influence of various agents transmitted to the embryo via the mother (Lutwak-Mann, 1954, 1962; Lutwak-Mann, Boursnell, and Bennett, 1960 ; Adams, Hay, and Lutwak-Mann, 1961 ; Lutwak-Mann, Hay, and Adams, 1962). Because of its large size (3-4.5 $\mathrm{mm}$. average diameter, at $6 \frac{1}{2}$ days of gestation) and numerical availability (up to 15 embryos per animal), the rabbit blastocyst at the pre-implantation stage of gestation represents a most useful object of study. During the course of our work we have evolved, and used extensively, a histological technique whereby the blastocysts can be studied in their entirety in the form of flatmounts. In view of the general interest in methods of testing drugs, especially in relation to pregnancy, this technique for the examination of pre-implantation embryos may find a useful application as a relatively simple and rapid screening test.

To prepare flat-mounts, the free-lying blastocysts are removed from the uterus and fixed in methanol ; in this fixative they are stable for a considerable length of time. Under a stereoscopic microscope the fixed blastocyst is punctured at the abembryonic pole and the trophoblast is then torn with fine needles in such a way as to make a star-shaped preparation, with the embryonic disk at its centre. This is allowed to dry on to a cover-slip, on which it is subsequently stained, usually with haematoxylin. Fuller details of the method have been published elsewhere (Moog and Lutwak-Mann, 1958). This procedure permits examination of the intact cells of the various regions of the blastocyst in their correct topographical relationships. The flat-mount preparations form a permanent experimental record; our collection, available for immediate reference and comparison, numbers to date approximately 2,000 embryos. But the chief merit of the method is that it provides a quantitative expression of some properties of the embryos, such as the dimensions of entire blastocysts and of embryonic disks ; flat-mounts can also be used for mitotic counts, which are particularly easy to make in the abembryonic region, where the trophoblast consists of a single layer of cells not yet lined by endoderm (Special Plate, Fig. 2).

Determinations of the mitotic index have proved illuminating in experiments with antimitotic substances -for example, demecolcine ("colcemid"). Atypical mitotic figures such as we have found, for example, in blastocysts from isoniazid-treated rabbits, are very easily seen in these preparations. Moreover. our observations extend to other features of the blastocysts-namely, the trophoblastic knobs, development of the embryonic disk including primitive streak formation, and outgrowth of extra-embryonic mesoderm. On this basis the developmental stage of the blastocysts at five to seven days of pregnancy can be assessed; details of these stages are found in our papers (Adams et al., 1961 ; Lutwak-Mann et al., 1962).

Gross inspection of the reproductive tract of treated animals as regards the number of corpora lutea in relation to embryos present, spacing of embryos, condition of the uterus, etc., is important in assessing the effect of treatment and should be carried out by a trained biologist. Preparation and staining of the flatmounts could probably be entrusted to a technical assistant skilled in the use of a dissecting microscope. To avoid bias and obtain dependable results our own histological examinations have routinely been made on coded batches of fixed blastocysts. The histologist's report was submitted to the research worker in charge of animal experimentation and considered in conjunction with observations made on the pregnant animal during treatment and with findings recorded at necropsy. If necessary, the blastocysts were re-examined in the light of full information pertaining to the animal's treatment and behaviour during the experimental period,

\section{LEGENDS TO SPECIAL PLATE}

The Plate shows representative embryos from untreated (control) and treated rabbits; although variations occur both within and between litters, those selected are considered typical for the treatment indicated. The day of mating is call-d day 0 of gestation. Average weight of experimental an mals, $4 \mathrm{~kg}$. Preparations were stained with Delafiely's haematoxylin All Figs. $\times 56$, except Fig. $2(\times 600)$.

Fig. 1.-Embryonic disk from an untreated rabbit. It is in the stage most frequently found $6 \frac{1}{2}$ diys after mating. The posterior end, where the disk is g:owing out pitor to the formation of the primitive streak, is marked with an arrow.

FIG. 2.-Single-layered trophoblast from the abembryonic pole of a 61-day blastocyst recovered from an unt:eated rabbit. In this region the cell boundaries are unteated rabbit. In this region the cell boundaries are
very distinct. Several dividing cells can be scen, and sex chromatin (arrowed) is visible in some of the resting nuclei of th:s female embryo.

FIG. 3-Degenerate remains of the embryonic disk of a $6 \frac{1}{2}$-day blastocyst, recovered from a rabbit treated parenterally on days 4 and 5 of gestation wi.h $50 \mathrm{mg}$. 6-mercaptopurine per kg. body-weight. The trophoblasts of these embryos (only partly shown) were much less affected.

Fig. 4.-Embryonic disk of a $6 \frac{1}{2}$-day blastocyst recovered from a rabbit treated parenterally on day 5 with $2.5 \mathrm{mg}$. E 39 soluble per $\mathrm{kg}$. body-weight.

FIG. 5.-Embryonic disk of a $6 \frac{1}{2}$-day blastocyst recovered from a rabbit treated orally on days 3,4 , and 5 of gestation with $0.5 \mathrm{~g}$. thalidomide per animal daily. The disk is degenerate and has an irregular outline. Signis of degeneration or marked retardation were seen in all the $306 \frac{1}{2}$-day embryos from three thaldomideteeated rabbits.

Fig. 6.-Embryonic disk of $6 \frac{1}{2}$-day blastocyst recovered from a rabbit treated orally on days $0-5$ of gestation with $100 \mathrm{mg}$. phthaloyl-DL-isoglutamine per animal daily. The disk is much larger than normal (compare with Fig. 1, at same magnification), although it is at the same stage of development.

Fig. 7.-Embryonic disk of a $6 \frac{1}{2}$-day blastocyst recovered from a rabbit treated parenterally on day 4 of gestation with $1 \mathrm{mg}$. oestradiol benzoate per animal The primitive streak has formed and extra-embryonic mesoderm (arrowed) is beginning to grow out from the posterior end of the disk. This stage is not normally reached until day 7 . 
CECILIA LUTWAK-MANN AND MARY F. HAY: TESTING OF EMBRYOTROPIC AGENTS

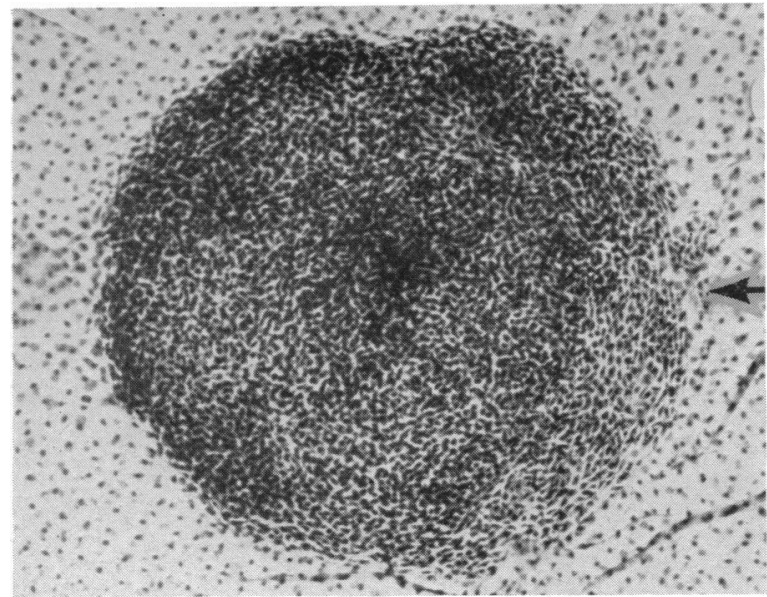

FIG. 1

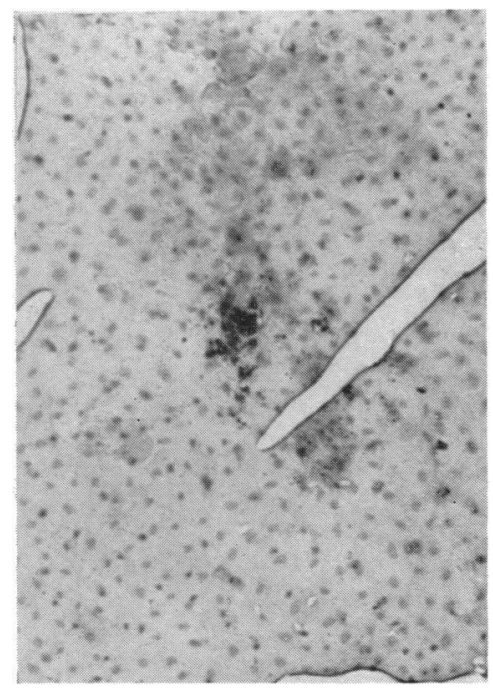

Fig. 3

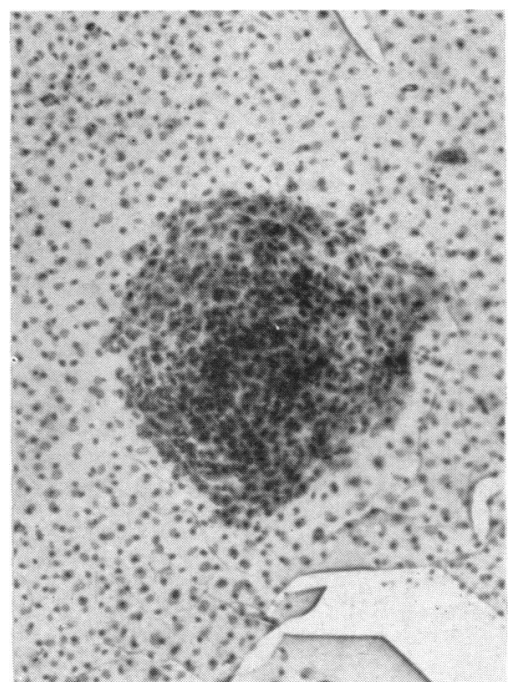

FIG. 4

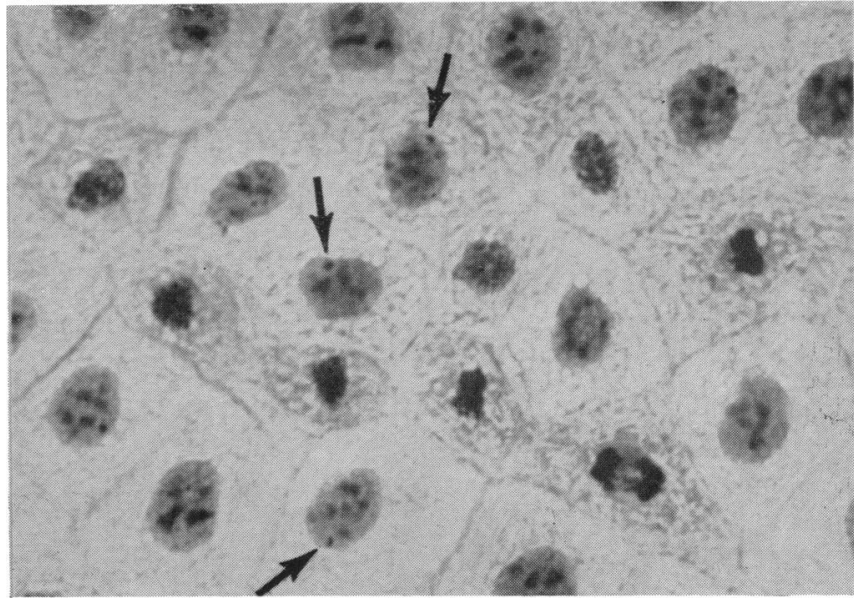

Fig. 2

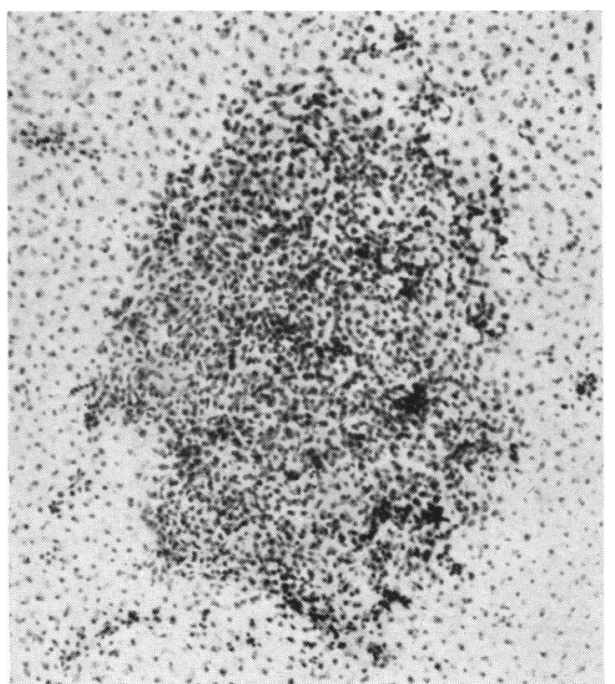

FIG. 5

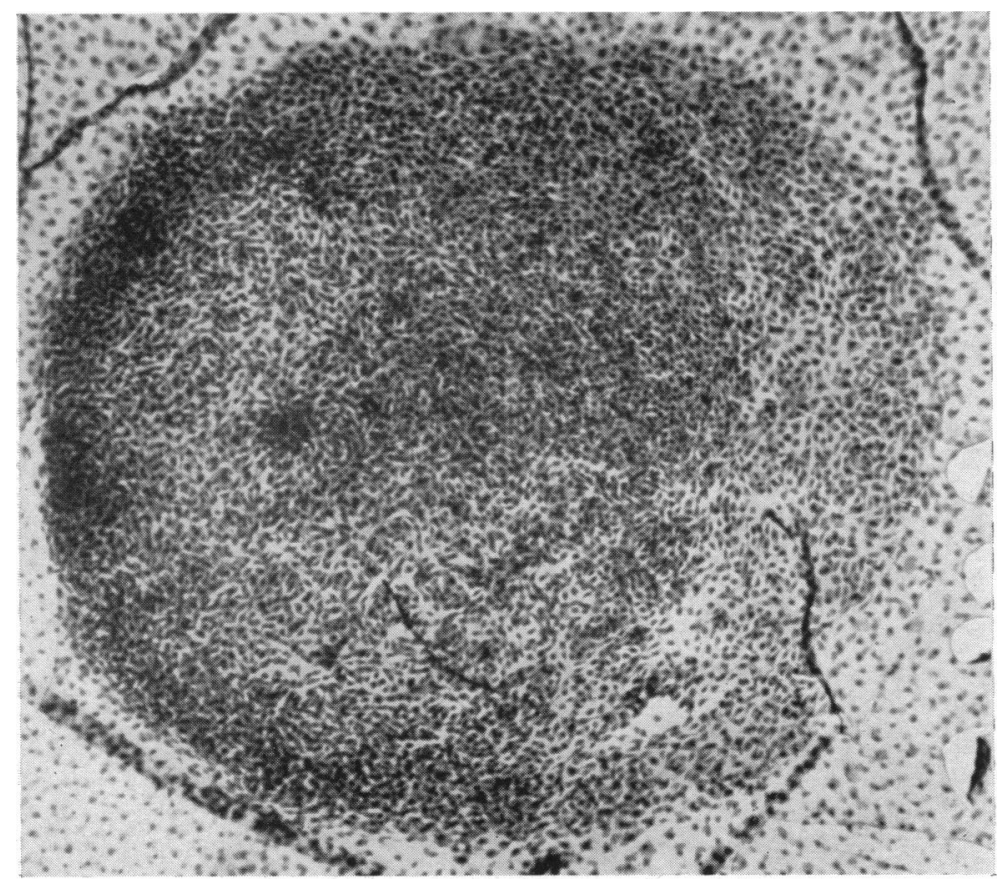

FIG. 6

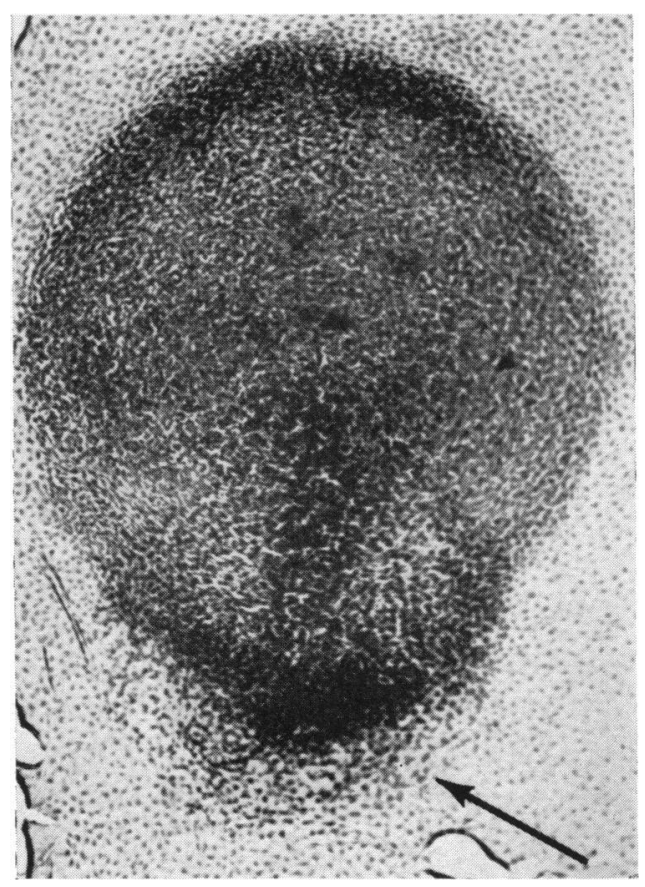

FIG. 7 
and in comparison with other litters from similarly treated rabbits. From a combination of these various studies the overall effect of the treatment was assessed.

Results with blastocysts from normal untreated rabbits of high fertility have brought out the existence of fairly wide limits of morphological variability, both between individual members of a litter, as well as between different but chronologically identical litters. These findings are of practical significance as they stress the need for the use of a sufficiently large number of polytocous laboratory animals, in either morphological, chemical, or pharmacological studies on embryos. We have found the route of administration of relatively less importance than the timing of treatment. With certain agents the results could be significantly modified by alterations in dose-timing, whereby, for instance, consecutive small amounts of a drug would induce in the embryos a response markedly different from that due to a single large dose.

Whenever there was an indication of a drug having exerted an effect upon the blastocysts, even if it involved not more than a general retardation of embryonic growth, the study was extended to include implantation and stages up to parturition. We have found that after certain treatments, gestation in the rabbit might be prolonged sometimes by several days, the litter being born dead. In other instances, litters from treated animals were born alive and at the expected time, only to die within a week, perhaps owing to innate weakness or a failure of lactation. The does were frequently re-mated after parturition, to test whether any treatment had permanently affected the animal's reproductive ability. Long-term impairment of ability to carry litters to term resulted, for example, from treatment of rabbits with small amounts of tretamine, an alkylating agent very toxic to the rabbit.

Among the substances that we have examined to date are several currently used in medical practice. These include certain ovarian, adrenal, and pituitary hormones; colchicine derivatives (colcemid, thiolcolciran); alkylating agents (thiotepa, mannomustine dihydrochloride ("degranol "), E 39 soluble*); a purine analogue (6-mercaptopurine); sulphonamides (methazolamide ("neptazane"), dichlorphenamide ("daranide "), carbutamide) ; cycloserine (oxamycin) ; salicylic acid ; iproniazid ; isoniazid ; thalidomide ("distaval ") ; and phthaloyl-DL-isoglutamine (a major metabolite of thalidomide in the rat and rabbit and also in man). We have also studied the effect of certain vitamins (vitamin $B_{12}$, pyridoxine, pantothenic acid, vitamins $A$ and $E$ ) administered in massive doses, and vitamin antagonists (aminopterin, amethopterin; analogues of vitamin $B_{12}$ ). In a limited number of experiments we have studied the effect of maternal exposure to $x$ rays.

Hitherto most of our work has been concerned with embryotropic agents which, when administered in doses that ostensibly did not affect maternal health, were nevertheless embryotoxic. Thus, for instance, colcemid, thiotepa, 6-mercaptopurine, E 39 soluble, and thalidomide all caused degeneration in blastocysts, predominantly in the area of the embryonic disk, but for each drug the histological changes were of a different and characteristic type. Some are illustrated in Figs. 3,4 , and 5 , together with a normal embryonic disk from an untreated rabbit (Fig. 1). At effective dose-levels of these drugs each embryo in any one litter was affected

*2 : 5-bis-ethylene-imino-3 :6-bismethoxyethoxybenzoquinone. C to some extent; with colcemid, 6-mercaptopurine, and E 39 soluble the damage was severe in all blastocysts, whereas with thiotepa and thalidomide there was a bigger range in the degree of degeneration.

For some time, however, our attention has been turned to certain phenomena noted in blastocysts from animals treated with substances of widely differing chemical nature, which included certain vitamins, hormones, and drugs. These changes manifested themselves severally as (1) an overall stimulation in which the blastocysts were generally advanced for their age (vitamin A), or were particularly uniformly developed, with no "backward " members in the litter (pantothenic acid); (2) embryonic disks that were disproportionately large for the entire diameters of the blastocysts, and also large for their developmental stage (phthalyl-DLisoglutamine (Fig. 6); $\alpha$-tocopherol alcohol ; cycloserine; growth hormone); (3) blastocysts of a size normal for their age but possessing embryonic disks that were developmentally advanced (oestradiol benzoate (Fig. 7) ; pregnant mares' serum). For the present we reserve our judgment on whether and to what extent these remarkable changes are ultimately beneficial and qualify for the definition of being embryo-" trophic." But we are inclined to think that a disproportionate stimulation and enhancement of growth of one region of the young embryo relative to the rest may be as significant a causative factor in embryonic death or malformation as are the more obvious degenerative effects of the frankly embryotoxic agents.

Taken together our findings serve to underline a fundamental fact, probably equally valid for the human species, but not always duly recognized-namely, the susceptibility of the pre-implantation embryo (and that includes the zygote in the oviduct) to agents passed on from the maternal organism.

Rabbit blastocysts can be successfully maintained in vitro, for periods up to 24 hours and longer (LutwakMann et al., 1962 ; current studies by M. F. H.) ; we envisage the culture of rabbit blastocysts coupled with the flat-mount technique as an additional and promising test-object for the study of growth-modifying and chemotherapeutic substances, also cytopathogenic agents such as viruses.

\section{Summary and Concluding Remarks}

A relatively simple and rapid histological technique is outlined for studying the action upon the pre-implantation rabbit embryo (blastocyst) of maternally transmitted agents.

The method consists in preparing flat-mounts from rabbit blastocysts recovered from pregnant animals that have received various treatments, and comparing them with embryos from normal untreated rabbits, partly on a quantitative basis. This has been more fully described in earlier papers, which should be consulted for technical details and discussion of the findings obtained with a fairly large number of embryotropic agents.

Examples of typical results obtained by the flat-mount technique are illustrated by photomicrographs showing the changes in the embryonic disk, produced as an outcome of treatment of the pregnant animals with 6-mercaptopurine, E 39 soluble, thalidomide, and phthaloyl-DL-isoglutamine, administered in each case in doses that did not affect maternal health.

While we are aware of the existence of embryotropic agents which are either specifically "antizygotic" or else 
exert their influence only after implantation, we none the less envisage the possibility of the flat-mount technique, when further perfected particularly in its quantitative aspects, becoming a valuable adjunct to other drugtesting and screening procedures, both generally and in relation to pregnancy.

We are indebted to Dr. G. F. Somers (The Distillers Co. Ltd.) for gifts of thalidomide and phthaloyl-DL-isoglutamine.
REFERENCES

Adams, C. E., Hay, M. F., and Lutwak-Mann, C. (1961). J. Embryol. exp. Morph." 9, 468

Lutwak-Mann, C. (1954). Ibid., 2, 1.

(1962) Nature (Lond.), 193, 653.

Boursnell, J. C., and Bennett, J. P. (1960). J. Reprod. Fertil., 169.

Hay, M. F., and Adams, C. E. (1962). J. Endocr., 24, 185 .

Moog, F., and Lutwak-Mann, C. (1958). J. Embryol. exp. Morph., 6, 57.

\section{BLOOD GROUPS AND DISEASE}

\section{ABH ANTIGENS ON hUMAN DUODENAL CELLS}

BY

\section{W. K. COWAN, M.D., M.R.C.P.Ed.}

\section{Clatterbridge General Hospital, Bebington, Cheshire, and Department of Medicine, University of Liverpool}

\section{[With Special Plate]}

Although the relationship between blood group $O$ and duodenal ulcer was first pointed out by Aird et al. (1954), its significance remains completely unknown. However, in the years since the original publication further work has both amply confirmed the association and has examined various hypotheses put forward to explain it. The theory of racial stratification which supposes that there may be a stratum of society, such as the Scots, where the incidence of both blood group $\mathrm{O}$ and duodenal ulcer is relatively high is still under investigation (Clarke, 1961), but racial stratification becomes less and less a possibility as further work continuing to show an association between blood group $\mathbf{O}$ and duodenal ulcer is reported from centres all over the world, embracing populations with different blood-group distributions.

Two hypotheses to explain peptic ulcerogenesis followed the finding of the firm relationship between duodenal ulceration and non-secretion (Clarke et al., 1956). First, it seemed that the blood-group antigens present in the subject's secretions might be able in some chemical or even physical way to protect the duodenal mucosa from ulceration. Such evidence as there is argues against such a suggestion (Clarke et al., 1959 ; McConnell, 1959 : Doll et al., 1961), yet it still remains a possibility. Second, the blood-group substances might be protective in an immunological way.

An immunological hypothesis has in fact been put forward by Cain (1957). He suggested that blood-group agglutinins found in leguminous plants (lectins) and swallowed in food might be ulcerogenic. In secretors this damaging effect would be neutralized by the bloodgroup antigens in the saliva and gastric juice. The hypothesis has lost some of its force following the work of Lawler (1957), who showed that many of these plant agglutinins are inactivated by heating: but the theory remains an attractive one. If indeed these agglutinins possess ulcerogenic properties, then the effect would probably be influenced by whether or not there were antigen sites on the mucosal surface cells with which they could combine or react. It therefore seemed worth while to investigate whether duodenal cells did in fact possess antigen sites, and, if so, whether there was any difference between the amount of blood-group substances as between secretors and non-secretors.
The blood-group antigen distribution in human duodenal mucosa has been investigated by Glynn et al. (1957), who used rabbit antisera and the fluorescent antibody technique. In the duodenum of group A and B secretor subjects, group antigen was found mainly in the goblet cells of the superficial epithelium, the columnar cells staining only faintly. A thin line of fluorescence outlined the lumina of the crypts indicating goblet-cell secretions coating the whole epithelial surface, and Brunner's glands were stained brightly. This situation contrasted with that in non-secretor subjects where no staining of the villi or crypts could be seen but where Brunner's glands nevertheless stained as brightly as in secretor subjects. The presence of $\mathbf{H}$ substance was not so predictable, since it was occasionally absent in the superficial mucosal zone in either secretor or non-secretor patients of either group $\mathbf{O}$ or A.

Szulman (1960) used conjugated human immune antisera to investigate the distribution of $A$ and $B$ antigens, and his findings in the duodenum confirm those of Glynn and his colleagues; he also noted antigen-loaded mucus welling up to the surface from Brunner's glands even in non-secretors. Selsnick (1959) examined human duodenal cells by both the fluorescent antibndy and the mixed-cell agglutination techniques and failed consistently to find blood-group antigen in the duodenal mucosal cells of non-secretor subjects by either method. Thus the consensus of evidence is that $\mathrm{ABH}$ antigens are not demonstrable on human duodenal cells of nonsecretor subjects. The present study was undertaken to confirm Selsnick's findings using mixed-cell agglutination, a more sensitive technique than the fluorescent antibody method. Nearly four times as many patients have been studied by this method. and the controls have been different from those in Selsnick's project.

\section{Material and Methods}

Specimens of fresh human duodenum were secured at gastrectomy, and in each instance a paraffin block of the tissue confirmed that it was in fact duodenum. Suspensions of duodenal mucosal cells, obtained by agitating pieces of mucosa in a solution of oxidized ascorbic acid (Cowan, 1962), were stored in $20 \%$ glycerol in normal saline at $-20^{\circ} \mathrm{C}$. 